\title{
QoS-GUARANTEED MODEL RESEARCH FOR A SATELLITE NETWORK
}

\author{
Chundong She, ${ }^{*}$ Junfeng Wang, ${ }^{* *}$ Ligang Fei, ${ }^{* * *}$ Kaiming Liu, ${ }^{*}$ and Shaohua Liu*
}

\begin{abstract}
As the backbone of transmission networks, satellite networks need to support a variety of services to meet the demands of new businesses and functions. Because of the characteristics of the satellite network, the services provided to users are also divided into multiple priorities. Thus, the quality of service (QoS) requirements for information in the satellite network will be more stringent. To this end, the space-based QoS-guaranteed model should have strong expansibility. Based on an analysis of the performance evaluation standards of QoS systems in a satellite network, this paper proposed a comprehensive evaluation criterion, and the simulation results show that this criterion could guarantee the QoS for various businesses in the satellite network.
\end{abstract}

\section{Key Words}

Satellite network, QoS, differentiated service

\section{Introduction}

The topology of the satellite network would change constantly. But, its energy is very limited, comparing with fixed wired networks and traditional cellular networks. Thus, the question of how to provide QoS support to a satellite network environment is currently a significant challenge [1]-[3]. In addition, satellite platforms often rely on battery power, which makes them susceptible to security attacks, especially with the increase in the number of networks [4], [5]. Providing QoS support in the satellite networks is a big challenge with many major problems [6]-[10]. Because the nodes in satellite networks belong to the wireless medium, the transmission of wireless signals is subject to the path loss and multiple path fading. The mobility of the nodes would lead to dynamic changes in the network topology and make the network state information changing frequently [11], [12]. It, in turn, makes routing

* School of Electronic Engineering, Beijing University of Posts and Telecommunications, Beijing 100876, China; e-mail: shurcd@vip.sina.com, \{kmliu, liushaohua\}@bupt.edu.cn

** School of Aeronautics and Astronautics, Sichuan University, Chengdu 610065, China; e-mail: wangjf@scu.edu.cn

*** Beijing Space Information Relay Transmission Technology Research Center, Beijing 100876, China; e-mail: feifrank@ 163.com

Corresponding author: Chundong She

Recommended by Dr. Xiaonv $\mathrm{Hu}$

(DOI: 10.2316/Journal.206.2018.5.206-0064) maintenance more difficult [13]-[15]. The mobile nodes in such a network use solar batteries. Thus, the consumption of energy must be carefully taken into account [16]-[18].

As we could see, the QoS guarantee for the satellite network is a complicated and systemic problem. Each layer within the protocol stack needs to provide a corresponding mechanism, which requires a new QoS mechanism designing and researching [19]-[22]. At present, there are few examples about QoS guarantee in the satellite network. Some researchers proposed the shortest path tree [23]-[26]. The call admission control strategy using the IntServ model [27], [28] was proposed by De Rango et al. An effective application of the DiffServ conversion [29] was proposed by Fantacci et al. The end-to-end QoS guarantee mechanism in the GEO satellite network [30] was proposed by Molinaro et al. Most of above research works did not consider QoS from a global perspective, which makes the model useless for satellite network information transmission.

\section{QoS Evaluation Standard in the Satellite Network}

Effectiveness and fairness are addressed by the QoS performance evaluation of a satellite network. As the user views, effectiveness refers to the service quality problems. But, from the systemic point of view, effectiveness generally refers to the service quantity problems. However, the service quality and the service quantity are often contradictory. Fairness is relative, and it is related to the balance between the users' requirements and the system state.

\subsection{Effectiveness Evaluation}

The normalized service quality function could be described as (1):

$$
\varphi_{i, j}(t)=\frac{\psi_{i, j}(t)}{\psi_{i, j}}
$$

$\psi_{i, j}$ is the target value of a certain service quality indicator, and $\psi_{i, j}(t)$ is the actual value at time $t$.

For the satellite network, main QoS indicators include the transmission delay and packet loss rate. In the general case, $\psi_{i, j}(t)>\psi_{i, j}$, so $\varphi_{i, j}(t)<1$, and users could not get satisfactory service. Furthermore, the performance of the network did not rely on a single service quality indicator. 
Different users might have different preferences for a certain performance or various performances. As a result, it needs more comprehensive attention. Here, one feasible way is to use the weighted average.

$$
\varphi_{i, j}(t)=\sum_{k=1}^{n} \omega \times \varphi_{(i, j), k}(t)
$$

$\omega_{k}$ is the weight of the QoS indicator function, and $\sum_{k=1}^{n} \omega_{k}=1$.

\subsection{Evaluation of Fairness}

After using a representative way as normalized performance function, the fairness of the flows could be judged by this function. Namely, if two normalization performance functions are equal, it means fairness. When treating the class of multiple flows as a whole, to assess its fairness, following two mechanisms are applicable:

(1) using the mean and variance: the fairness coefficient of all the flows in class $i$ is defined by (3):

$$
\eta_{\delta, i}(t)=\sigma_{\delta_{i}}(t) / \delta_{i}(t)
$$

where $\delta_{i}(t)$ is the total performance function of all the flows, and $\sigma_{\delta_{i}}(t)$ is the variance. The smaller $\eta_{\delta, i}(t)$ represents the smaller performance difference and greater fairness.

(2) The fairness index based on Raj Jain's approach

Based on the assumption that fairness means the values of the normalized performance functions are equal, we can obtain the fairness index of all flows with respect to class $i$ :

$$
f_{\delta, i}\left(\delta_{i, 1}, \delta_{i, 2}, \ldots, \delta_{i, n}\right)=\left(\sum_{j=1}^{n} \delta_{i, j}\right)^{2} / n \sum_{j=1}^{n} \delta^{2}
$$

When all $\delta_{i, j}$ are equal, this means that the fairness of class $i$ is the best.

\subsection{Comprehensive Performance Evaluation Criterion}

The above analysis is based on some certain performance, but high requirements in one performance area may lead to a serious decline and will not be accepted by users. Some kind of network transmission control strategy could be carried out through compromising on multiple areas of quality in the service requirements.

The final goals in performance researches on network transmission control include efficient performance overall and the QoS requirements. Network performance parameters, such as overall efficiency, could reflect and measure these goals. Because most of the QoS requirements demand the delay and loss rate to be as small as possible, we could exactly choose the delay $D$ as well as the loss rate
$L$ as the required parameters for QoS. In the following sections, we give two comprehensive performance evaluation mechanisms.

Criterion 1: throughput $T+$ delay $D$

The power equation is proposed to evaluate the effectiveness and define the relationship between the throughput and the delay.

$$
\text { Power }=\frac{\text { Throughput }^{a}}{\text { Delay }}(0<a<1)
$$

However, it is not appropriate to use such formula. This formula is based on the M/M/1 queue model, and not suitable for the satellite network. Moreover, this formula is defined in a single connection. Consider that a multiqueue model is composed of multiple service classes. That is to say, if the length of the queue is limitless, the lengths of all have the same distribution, and the server works persistently, then the systematic total average delay is defined by (6):

$$
\bar{d}(\lambda)=\frac{1}{\lambda} \sum_{i=1}^{N} \lambda_{i} \overline{d_{i}}
$$

where $\lambda_{i}$ represents the average arrival rate of packets of service class $i, \bar{d}$ represents the average queueing delay of packets of service class $i$, and the contribution to the average system delay of service class $i$ is $\frac{\lambda_{i}}{\lambda} \overline{d_{i}}$.

Thus, the original power formula changes to (7):

$$
\text { Power }=\frac{\text { Throughput }^{a}}{\bar{d}(\lambda)}=\frac{\left(\sum T_{i}\right)^{a}}{\frac{1}{\lambda} \sum \lambda_{i} \overline{d_{i}}}(0<a<1)
$$

We can see from such formula that to enhance the effectiveness of the cyber source distribution, we need to make (7) gain greater value. Figure 1 shows this power curve of the network, and in theory, the distribution strategy should summit the curved peak.

For a satellite network, however, it is very difficult to accurately control achievement of an absolute peak. As a matter of fact, we could only roughly change a certain load and the arrival speed of packets so as to make the effectiveness of the network on the area approach the curved peak, which enhance the system's performance. The relationship between the system packet loss rate and

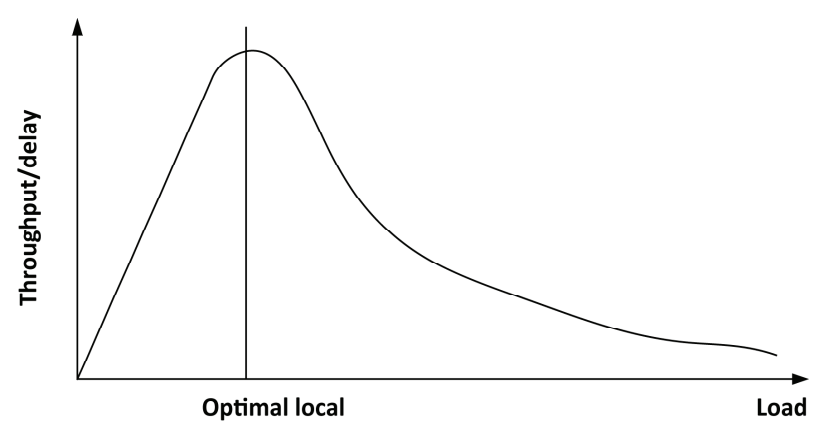

Figure 1. Schematic diagram of the power formula. 
throughput is similar. According to above equation, we could define the mean formula as (8) for the packet loss rate:

$$
\bar{l}(\lambda)=\frac{1}{\lambda} \sum_{i=1}^{N} \lambda_{i} \overline{l_{i}}
$$

where $\overline{l_{i}}$ represents the packet loss rate of some service class $i$.

Thus, in a similar way, a similar power formula is defined as infra:

$$
\text { Power }=\frac{\text { Throughput }^{a}}{\bar{l}(\lambda)}=\frac{\left(\sum T_{i}\right)^{a}}{\frac{1}{\lambda} \sum \lambda_{i} \overline{l_{i}}}(0<a<1)
$$

Similar to the above discussions, in a satellite network, we could also tune certain loads and the arrival speed of packets to make the effectiveness of the network on the area near the curved peak, which would improve the system's performance.

Criterion 2: quality of service requirements + fairness $F$

By just selecting the delay $D$ and loss rate $L$ as the required parameters for QoS, such criterion could be divided into following two criterions.

(1) Delay $D+$ fairness $F$

Considering that fairness is based on the queuing delay, we could use the queuing delay as the performance parameters for the proportional fairness principle. The delay measurement of the simplest service class is based on the average queuing delay over a long period of time. If we use $\overline{d_{i}}$ to represent the average queuing delay of a packet in the service class $i$, then the proportional fairness principle could be defined in 10:

$$
F=\frac{\overline{d_{i}}}{\delta_{i}}=\frac{\overline{d_{j}}}{\delta_{j}}(i, j=1,2, \ldots, N)
$$

where the parameter $\delta_{i}$ is the delay differentiation parameter required by the user, and $\delta_{1}>\delta_{2}>\ldots>\delta_{N}>0$. Assume that the proportional fairness principle is feasible, $\bar{d}(\lambda)$ is the total average queue delay, and the packet length distributions for all classes of services are the same, then this assumption implies that:

$$
\sum_{i=1}^{N} \lambda_{i} \overline{d_{i}}=\lambda \bar{d}(\lambda)
$$

Combining (10) and (11) together, the average queue delay of the service class $i$ is:

$$
\overline{d_{i}}=\frac{\delta_{i} \bar{d}(\lambda)}{\delta_{1} \frac{\lambda_{1}}{\lambda}+\delta_{2} \frac{\lambda_{2}}{\lambda}+\cdots+\delta_{N} \frac{\lambda_{N}}{\lambda}} \quad(i=1,2, \ldots, N)
$$

According to previous three formulas, we could draw following conclusions: (1) the average delay in the service class $i$ would increase with the increase in the

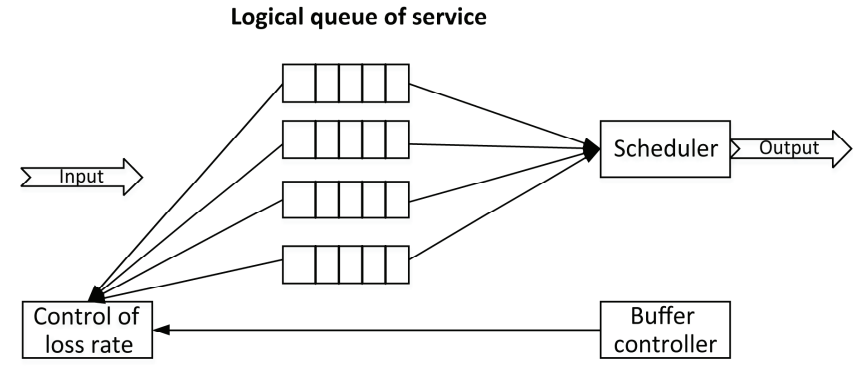

Figure 2. Network transmission control model for the $N$ service classes.

average arrival rate of the service class $j$. (2) Compared with the load of a lower class, the load of a senior class would have a greater impact on the increase in the average delay. (3) If the delay differentiation parameter of a certain service class increases, all other service classes' average delays would be reduced, and the average delay of this service class would increase. (4) When the partial load of service class $i$ is moved to the service class $j$, and the total load is unchanged, where $i<j$, each kind of average delay would increase.

(2) The loss rate of $L+$ fairness $F$

The network transmission control model of the $N$ service classes is illustrated in Fig. 2, where every logical queue corresponds to a certain service class.

The proportional fairness principle requires the performance proportional to those corresponding distinguishing parameters set by the network managers:

$$
F=\frac{\overline{l_{i}}}{\sigma_{i}}=\frac{\overline{l_{j}}}{\sigma_{j}} \quad(i, j=1,2, \ldots, N)
$$

where $\sigma_{i}$ is the loss rate distinguishing parameter, and $\sigma_{1}>\sigma_{2}>\cdots>\sigma_{N}>0$. The main idea of the proportional loss model is to keep the real-time loss rate estimation $l_{i}$ for every class constant relatively.

Criterions 1 and 2 should be independent of each other. They could be combined into an evaluation criterion for the satellite network to provide a more comprehensive performance evaluation.

\section{Satellite Network QoS Performance Evaluation}

We used the OPNET software to carry out the simulation.

\subsection{The Resource Reservation}

In contrast to adopt a confirm service business, the connection-oriented service business should be provided with the resource reservation protocol to ensure the QoS requirements on the network. Resource reservation could use the general signalling responses model. In this model, the bandwidth should be mainly taken into consideration.

(1) Resource reservation mainly involves reservation and cancellation. Reservation was for the resource reservation of a business request, where the label edge router 


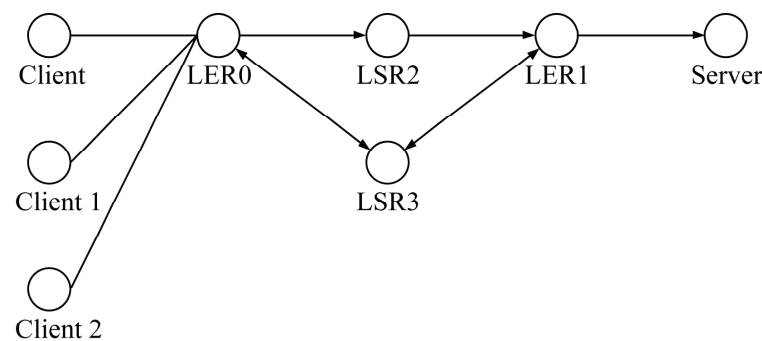

Figure 3. Schematic diagram of the simulation model for business reservation.

(LER) node sent a reservation signal to the downstream node. The downstream node judges whether the business had been reserved resources, and if it had, it would not be treated. Otherwise, the node should give judge. The LER node must receive all the nodes with a successful reservation response; otherwise, it returned the reservation failure response. Cancellation happened when a node was unable to achieve the resource reservation request, or the sending source needed to cancel the reservation application, or a routing switch, and all the nodes on the path, needed to be cancelled with resource reservation. The LER node sent a cancellation signal to each node. After receiving the signal, the downstream nodes released the reserved bandwidth and recorded the business that still has no resource reservation.

(2) The simulation results: there are three businesses sharing one link, two businesses without resource reservation, whose priorities are 0 and 1 , and the loss packet threshold value is 40,000 . There is one resource reservation service, whose priority is 3 , with a resource reservation of 40,000, a bottleneck link bandwidth of 100,000 (bit/s), a task flow rate of $40 \mathrm{p} / \mathrm{s}$, where each packet is 1,000 bits, and the added packet head is 1,152 bits in total. The simulation model is illustrated in Fig. 3.

For each resource reservation service, the nodes have a special sending queue, and each data packet is sent according to the reserved bandwidth. The network service quality is relatively stable. As illustrated in Fig. 4, the delay in the resource reservation service does not have a high fluctuation.

For the loss packet rate, the resource reservation service also has a certain loss, and the reason is that the reserved bandwidth is smaller than the client's sending bandwidth. Thus, the length of the corresponding sending queue on the satellite's sending port will make full use of the drop tail strategy. The loss packet rate is relatively higher and also influenced by the priority which means that a higher priority business's dropping probability is lower as illustrated in Fig. 5. We realized that the reservation mechanism was a kind of imperfect reward mechanism with packet loss, using the drop tail. Therefore, the resource reservation could not be lower than the transmission speed; otherwise, the discard mechanism is not ideal.

The resource reservation's throughput rate is very stable with respect to the throughput rate. However,

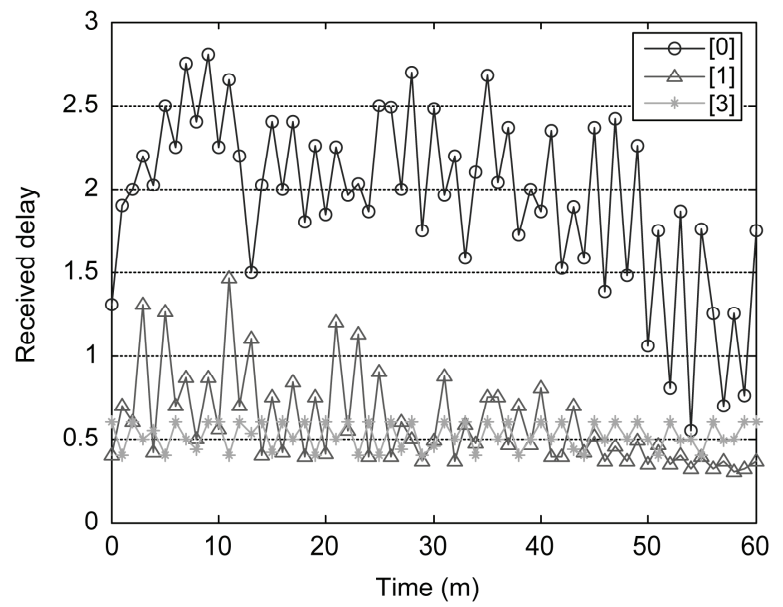

Figure 4. Comparison of the three kinds of delay.

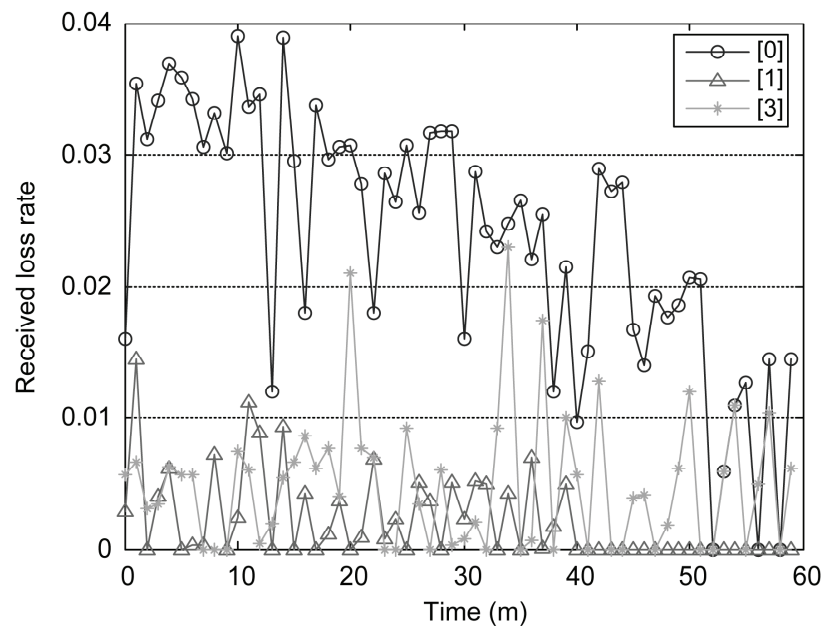

Figure 5. Loss comparison chart of resources.

those services without reservation will strive for bandwidth according to the priority (refer to Fig. 6).

\subsection{The Middle-Low Orbit Constellation Simulation}

The complex model considered the QoS effects of the middle orbit satellite, rather than considering the routing problem. The differences between middle orbit and low orbit are mainly due to delay and bandwidth. The middle orbit satellite's bandwidth is larger, whereas its delay is longer. Therefore, different choice of route between a middle orbit and a low orbit has significant effects on QoS. This model here used 66 low orbit satellites and 18 middle orbit satellites, as shown in Fig. 7.

Client $00,01,02$ is a low orbit service, respectively, passing through $2,3,4$ low orbit satellites to route. Client $10,11,12$ is a middle orbit service, respectively, passing through 1,2,3 middle orbit satellites and two low orbit satellites to route. From the utilization rate illustrated in Fig. 8, we could see that the link utilization rate was $25 \%$ without congestion happening. 
At this time, the transmission delay of six businesses is illustrated in Fig. 9.

In this case, the numbers of satellites were the same, the delay in transmitting completely through the low orbit satellites was smaller than that in the middle orbit, but they were also easily congested in case of heavy load. When some new user clients and client 00 shared one link, network congestion occurred and the delay of six businesses was illustrated in Fig. 10. At this time, the business delay depended entirely on the significant increase in transmission in the low orbit satellites, in contrast to the transmission in the middle orbit satellites. The low orbit satellite transmissions have no obvious advantage in heavy load conditions but could improve the QoS in case of less network load.

As for the throughput rate, low orbit satellites with limited resources and small bandwidth would easily reach saturation. However, transmissions through middle orbit satellites could still greatly improve the throughput rate, as illustrated in Fig. 11.

\section{Differentiator of received}

(bits) $\times 10^{4}$

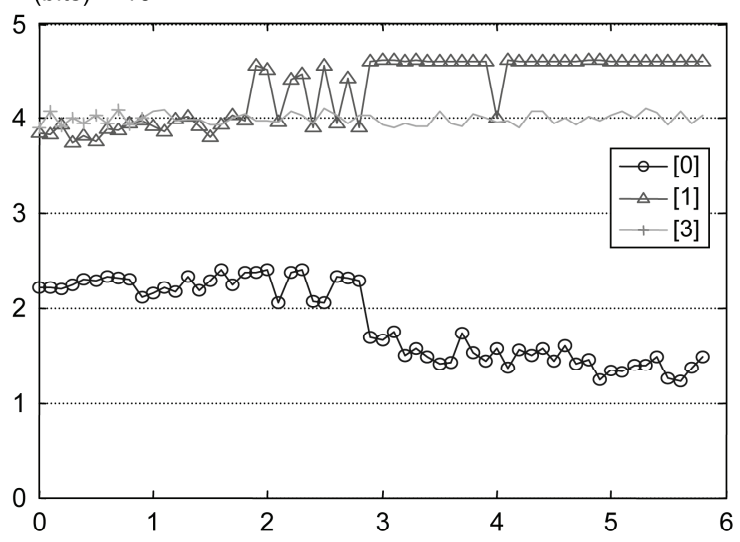

Figure 6. Schematic diagram of three situations.

\subsection{Satellite Network Priority Simulation}

For three confirm service businesses, with priority of $0,1,2$, respectively, sharing one link, 100,000 bits as the bottle-

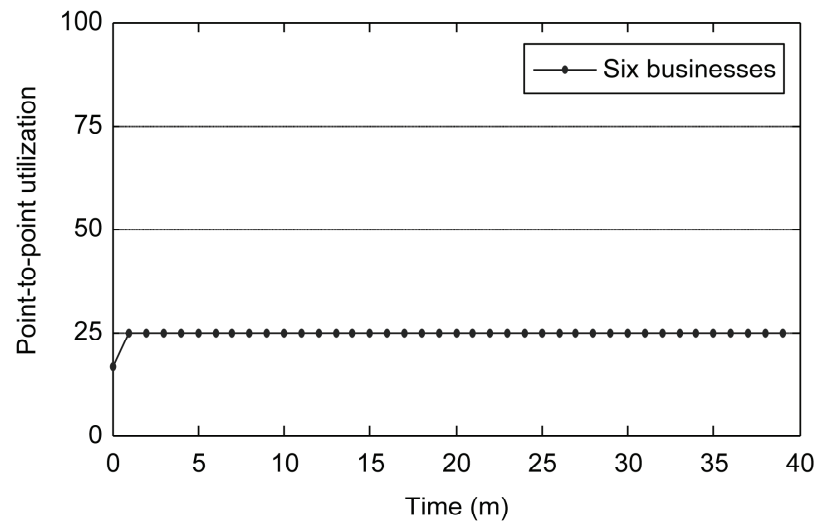

Figure 8. Schematic diagram of the link business without congestion.

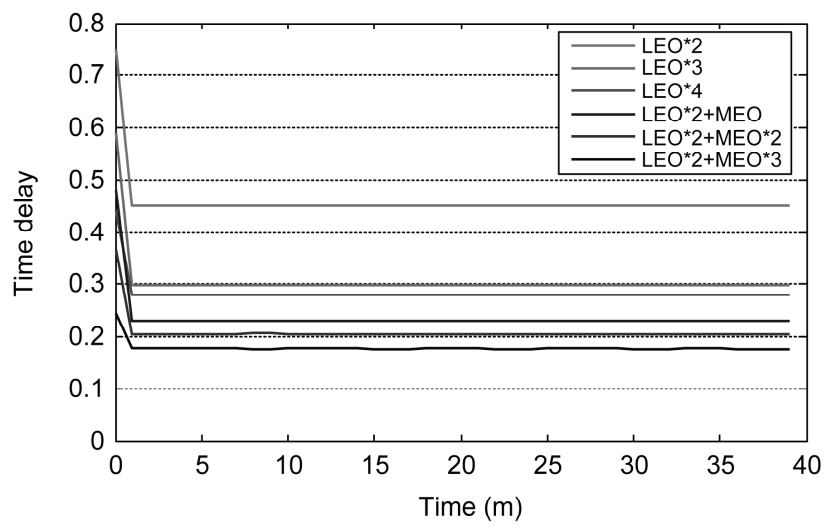

Figure 9. The delay comparison of six businesses in the low load condition.

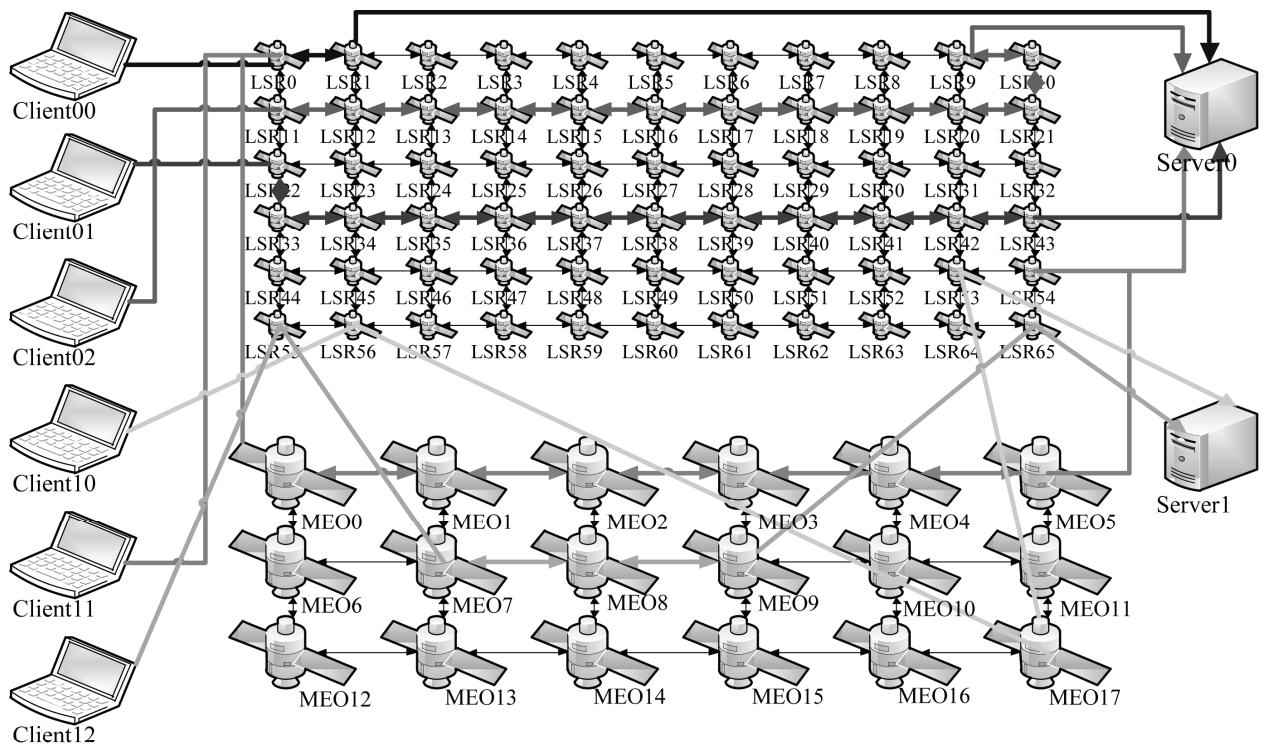

Figure 7. Schematic diagram of middle-low orbit constellation simulation. 


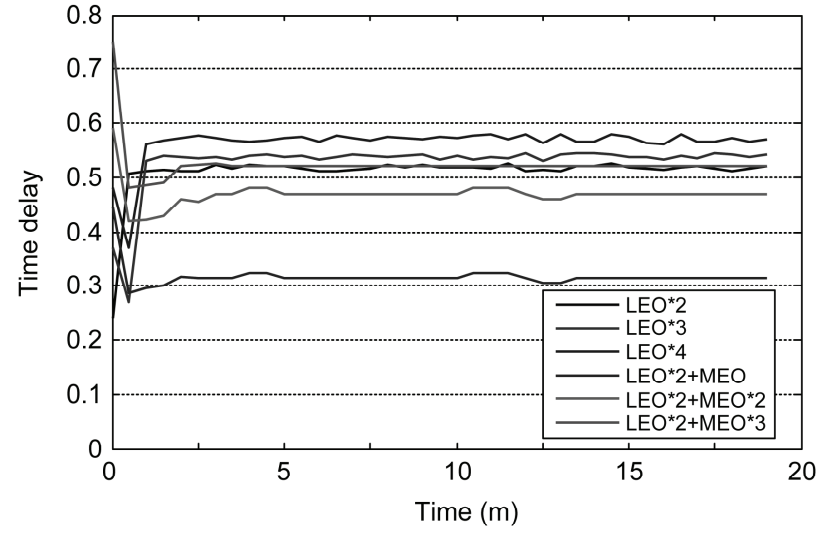

Figure 10. The delay comparison of six businesses in a large load condition.

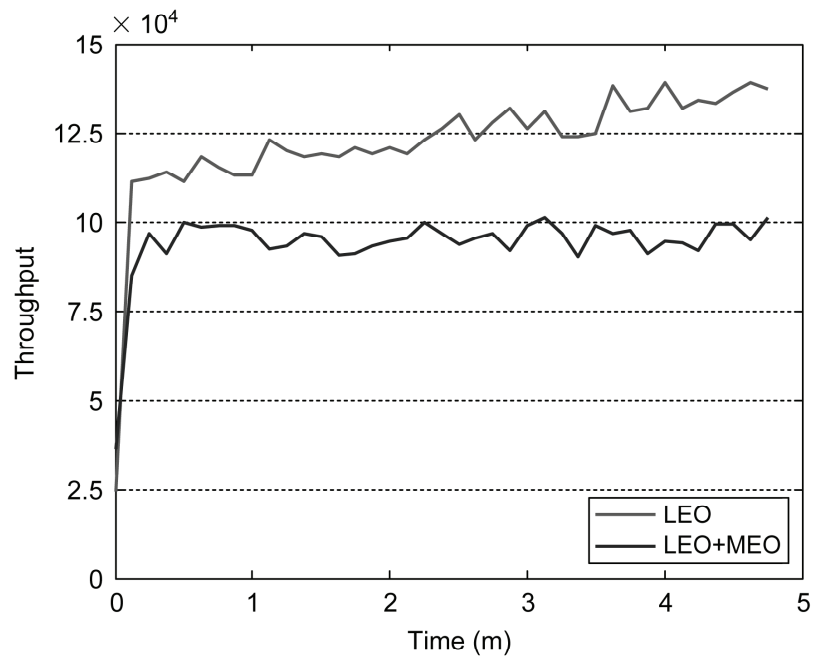

Figure 11. Comparison of middle-low orbit throughput rates.

neck link bandwidth, a task flow rate of 50 packets per second, each packet of 1,000 bits, and 1,152 bits of added packet head in total, namely, 57,600 bit/s, the total flow rate was $172,800 \mathrm{bit} / \mathrm{s}$, and 3,000 for the entire threshold for the packet loss. The degree of congestion was high relatively. For different levels of services, each satellite node had a different sending queue which weight in sending was different from the weight of the packet loss. Thus, the qualities of services were also different. There were three queue parameters: weight $[0]=1$, weight $[1]=2$, weight $[2]=3$. These parameters could be adjusted in accordance with real needs.

(1) The simulation results for delay: we could see that the delay with higher priority was small and stable relatively, due to the larger weight of sending, as illustrated in Fig. 12.

(2) The simulation results for the packet loss rate: we could see that the services with lower priority lost more packets as illustrated in Fig. 13. Many factors would affect the packet loss probability, and different priority

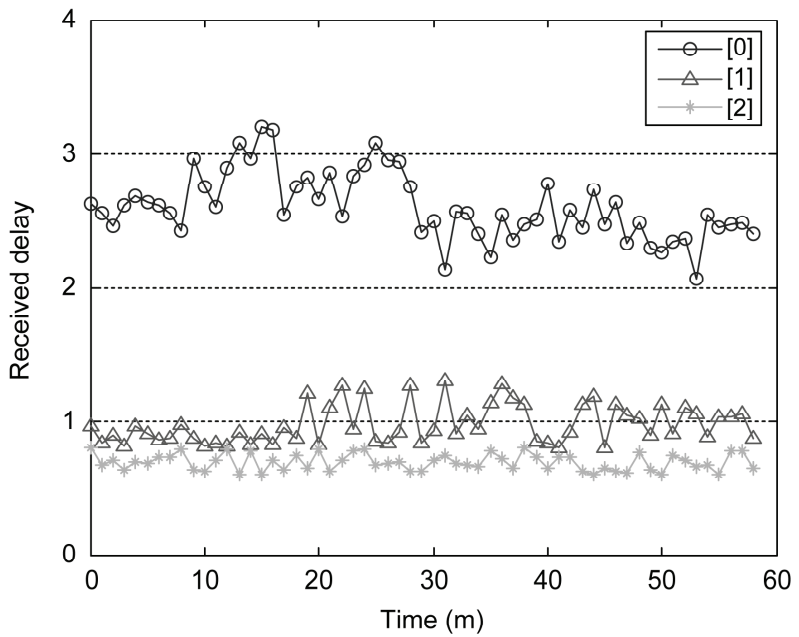

Figure 12. Delay comparison.

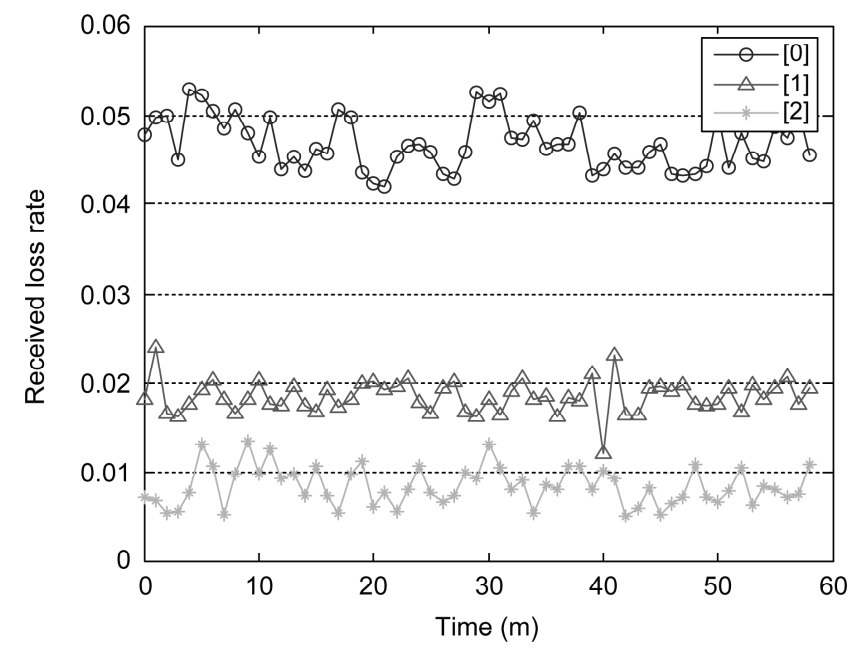

Figure 13. Comparison of the packet loss rate for the three kinds of businesses.

queues in the sending port of the satellite nodes had different packet loss probabilities. Each queue decided its own different packet loss probability according to the exp-domain of mpls tags. The exp-domain in such three kinds of services was the same, but when in different queues, only the influences of the different priority queues for packet loss were evident.

(3) The simulation results throughput: the factor which affects the throughput rate is the QoS. That is to say, the sending rate is determined by different sending weights of the different priorities. The throughput rate of high priority services was high, as illustrated in Fig. 14. The total throughput rate of the network was limited by the bandwidth of the bottleneck link, the fluctuation of the throughput rate related to the transmission control protocol (TCP) mechanism, and the throughput rate fluctuation was small relatively with resources reservation. 


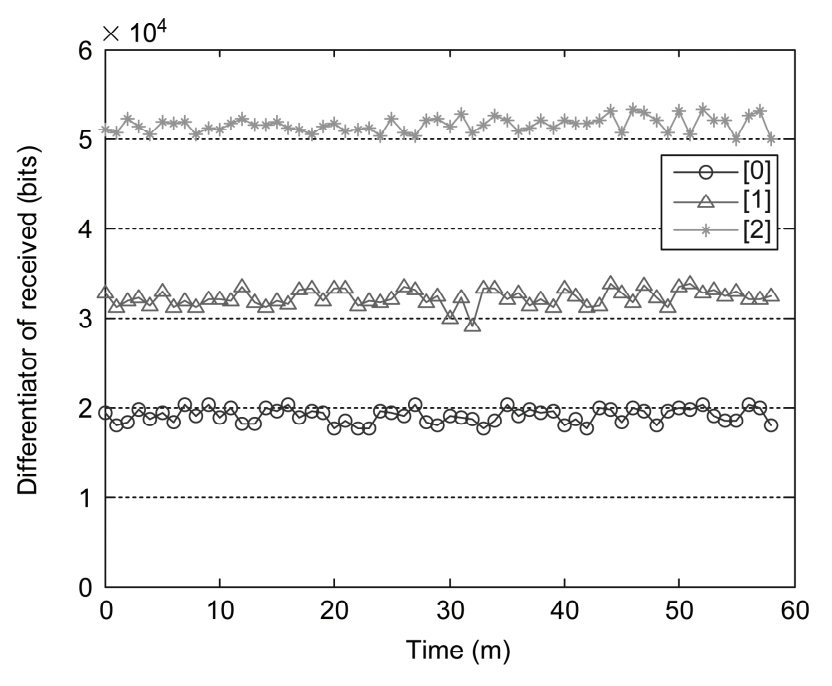

Figure 14. Comparison of the throughput rate in three kinds businesses.

\section{Conclusion}

With the advancement of mobile satellite networks, the technology for QoS assurance has become a research hot spot in this field. Guaranteeing QoS in satellite networks tends to be more complex and difficult to achieve because of the characteristics of satellite networks. In this paper, a standard on evaluating the performance of a satellite network is proposed, and the OPNET simulation software has been used to analyse the performance. Those simulation results show that such comprehensive evaluation method achieved a better result.

\section{Acknowledgement}

This research was partly supported by the National Natural Science Foundation of China (Grant Nos. 91438120, 91338107), the Ph.D. Program Foundation of the Ministry of Education of China (No. 20130181110095), the National Defense Equipment Advance Research Shared Technology Program of China (41402050301-170441402065), the Jiangsu Future Networks Innovation Institute Prospective Research Project on Future Networks (BY2013095-3-08), and Technological Equipment Mobilization Program of Dongguan (KZ2017-06).

\section{References}

[1] A. Destounis and A.D. Panagopoulos, Dynamic power allocation for broadband multi-beam satellite communication networks, Communications Letters, IEEE, 15(4), 2011, 380-382.

[2] G. Maral and M. Bousquet, Satellite communications systems: Systems, techniques and technology (John Wiley \& Sons, 2011).

[3] S.E. Burrowbridge, Optimal allocation of satellite network resources, 1999.

[4] F. Alagöz and G. Gür, Energy efficiency and satellite networking: A holistic overview, Proceedings of the IEEE, 99(11), 2011, 1954-1979.

[5] C. Warty, Cooperative communication for multiple satellite network, 2010 IEEE Aerospace Conference, Big Sky, MT, USA, 2010, 1-7.
[6] L. Liu, D. Wang, L. Wang, Research of a new non-layer protocol architecture for satellite network, TELKOMNIKA Indonesian Journal of Electrical Engineering, 12(6), 2014, 4654-4663.

[7] J. Chen, L. Liu, and X. Hu, Towards an end-to-end delay analysis of ubiquitous access network, Science China Information Sciences, 56, 2013, 110101:1-110101:13.

[8] H. Liu, and F. Sun, A QoS-oriented congestion control mechanism for satellite networks, Mathematical Problems in Engineering, 2014(11), 2014, 1-13.

[9] K. Arshad, R. MacKenzie, U. Celentano, et al. Resource management for QoS support in cognitive radio networks, Communications Magazine, IEEE, 52(3), 2014, 114-120.

[10] E. Blasch, K. Pham, G. Chen, et al. Distributed QoS awareness in satellite communication network with optimal routing (QuASOR), 2014 IEEE/AIAA 33rd, Digital Avionics Systems Conference, Colorado Springs, CO, USA, 2014, 6C3-1-6C3-11.

[11] K.J. Birdwell, B. Moran, D. Feinleib, Multi-packet transport structure and method for sending network data over satellite network, U.S. Patent 6,172,972. 2001-1-9.

[12] J. Wenjuan and Z. Peng, QoS routing algorithm based on traffic classification in LEO satellite networks, 2011 Eighth International Conference on. IEEE, 2011, 1-5.

[13] L. Wood, A. Clerget, I. Andrikopoulos, et al., IP routing issues in satellite constellation networks, International Journal of Satellite Communications, 19(1), 2001, 69-92.

[14] H. Xu, F. Huang, and S. Wu, A distributed QoS routing based on ant algorithm for LEO satellite network, Journal of Electronics (China), 24(6), 2007, 765-771.

[15] A. Stentz, Optimal and efficient path planning for unknown and dynamic environments, International Journal of Robotics \& Automation, 10(3), 1993, 89-100.

[16] E. Lutz, M. Werner, and A. Jahn, Satellite systems for personal and broadband communications (Springer Science \& Business Media, 2012).

[17] N. Celandroni, F. Davoli, and E. Ferro, Static and dynamic resource allocation in a multiservice satellite network with fading, International Journal of Satellite Communications and Networking, 21(4-5), 2003, 469-487.

[18] F. Ganhão, L. Bernardo, R. Dinis, et al., Energy-efficient QoS provisioning in demand assigned satellite NDMA, 2012 21st International Conference on Computer Communications and Networks (ICCCN), Munich, Germany, 2012, 1-8.

[19] R. Mauger and C. Rosenberg, QoS guarantees for multimedia services on a TDMA-based satellite network, Communications Magazine, IEEE, 35(7), 1997, 56-65.

[20] Q. Liu, M. Zhao, and F. Lin, A QoS guarantee service serving model in LEO satellite networks based on differential game, Communications, China, 11(14), 2014, 128-134.

[21] R. Challoo and S. Dubey, Simultaneous state and actuator fault estimation with fuzzy descriptor PMID and PD observers for satellite control systems, International Journal of Robotics and Automation (IJRA), 2(5), 2011, 344.

[22] Y. Rao, J. Zhu, W. Chen, et al., Agent-based QoS routing for polar-orbit LEO satellite IP networks, Journal of Convergence Information Technology, 7(17), 2012, 197-206.

[23] R.I. Qureshi and O. Alphand, SIP-based QoS control over satellite networks, Proc. of the IEEE International Conf. on Emerging Technologies. Piscataway: IEEE Computer Society, 2005, 242-245.

[24] A. Bhattacharya and A. Kumar, A shortest path tree based algorithm for relay placement in a wireless sensor network and its performance analysis, Computer Networks, 71, 2014, 48-62.

[25] Q. Yanbin, C. Xingyuan, D. Xuehui, et al., Layered secure access research for space network, 2008 International Symposium on Computer Science and Computational Technology (ISCSCT), Shanghai, 2008, 307-311.

[26] A. Bhattacharya, and A. Kumar, A shortest path tree based algorithm for relay placement in a wireless sensor network and its performance analysis, Computer Networks, 71, 2014, 48-62.

[27] F. De Rango, M. Tropea, and S. Marano, Call admission control for integrated Diff-Serv terrestrial and Int-Serv satellite network, 2004 IEEE 59th Vehicular Technology Conference, 2004. VTC 2004-Spring, Milan, Italy, 2004, Vol. 5, 2921-2925.

[28] I. Mahadevan and K. M. Sivalingam, Quality of service architectures for wireless networks: IntServ and DiffServ models, 
Proc. Fourth International Symposium on Parallel Architectures, Algorithms, and Networks, 1999 (I-SPAN'99) IEEE, Perth/Fremantle, WA, Australia, 1999, 420-425.

[29] R. Fantacci, R. Gubellini, D. Tarchi, et al. An efficient DiffServ switch for satellite communication systems based on cellular neural networks, IEEE, 2004, 2716-2720.

[30] A. Molinaro, F. De Rango, S. Marano, et al., A scalable framework for end-to-end QoS assurance in IP-oriented terrestrialGEO satellite networks, IEEE Communications Magazine, 43(4), 2005, 130-137.

\section{Biographies}

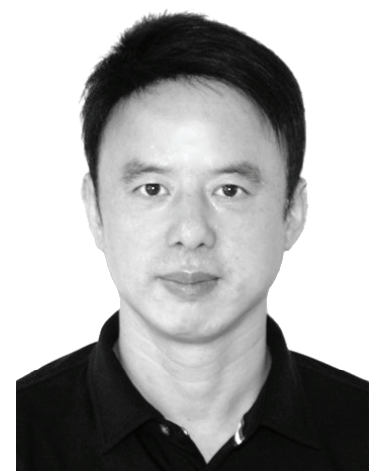

Chundong She born in 1971 is now a professor at the School of Electronic Engineering, Beijing University of Posts and Telecommunications, China. He received his B.Sc. degree in computer science and engineering from PLA University of Science and Technology, China, in 1991. Then, he received his M.Sc. degree in computer science from the Nanjing University of Aeronautics and Astronautics, China, in 1997. Later, he received his Ph.D. from the School of Computer Science and Engineering, at the University of Electronic Science and Technology of China, in 2004. His research interests mainly include wireless sensor network and information security.

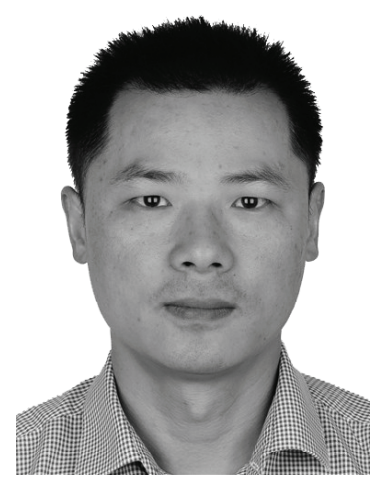

Junfeng Wang received his M.Sc. degree in computer application technology from Chongqing University of Posts and Telecommunications, Chongqing, China, in 2001. He received his Ph.D. in computer science from the University of Electronic Science and Technology of China, Chengdu, in 2004. Since July 2004 until August 2006, he had been a post doctor in the Institute of Software,

Chinese Academy of Sciences. Since 2006, he has been a professor at the School of Aeronautics and Astronautics and the College of Computer Science, Sichuan University, China.

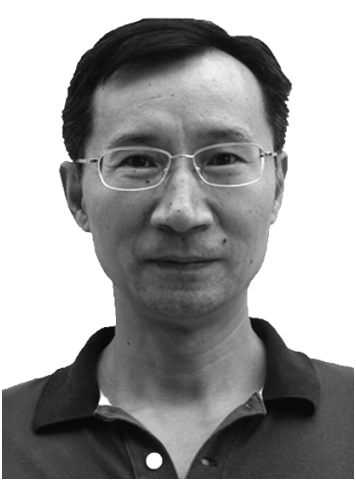

Ligang Fei received his B.S. degree from Tsinghua University, China. His current research interests include space information networks and satellite communications.

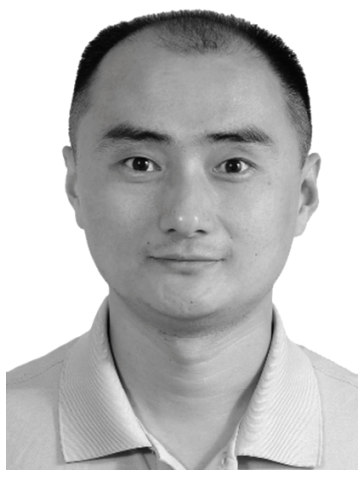

Kaiming Liu received his B.S. and Ph.D. degrees in communication engineering from the Beijing University of Posts and Telecommunications (BUPT), in 2001 and 2006, respectively. $\mathrm{He}$ is currently an associate professor at the School of Electronic Engineering, BUPT, and Director of the Wireless Smart Communications and Networking (WSCN) Laboratory. Currently, his major work areas include broadband telecommunications as well as heterogeneous wireless networks. He has published more than 70 papers on topics related to wireless communications and networking and applied for more than 20 patents.

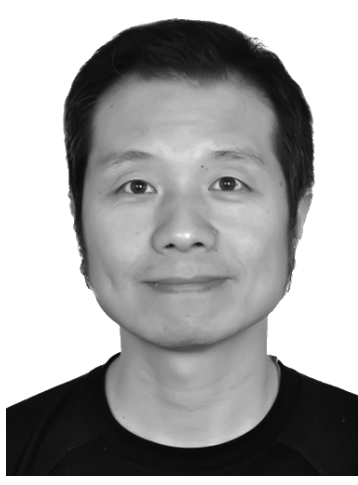

Shaohua Liu received his B.S. and M.Sc. degrees from Zhejiang University in 1998 and 2001, respectively. Then, he received his Ph.D. in computer science from Institute of Software, Chinese Academy of Sciences in 2006. $\mathrm{He}$ is now an associate professor at the School of Electronic Engineering, Beijing University of Posts and Telecommunications, China. His major interests involve telecommunications engineering and software engineering. 